\title{
A Target-Specific Electrode and Lead Design for Internal Globus Pallidus Deep Brain Stimulation
}

\author{
Xavier Vasques $^{\mathrm{a}-\mathrm{e}} \quad$ Laura Cif $^{\mathrm{a}-\mathrm{e}}$ Gérard Mennessier $^{\mathrm{f}}$ Philippe Coubes $^{\mathrm{a}-\mathrm{e}}$ \\ a Service de Neurochirurgie, CHRU Montpellier, bIGF, 'CNRS UMR5203, dINSERM U661, ' Université Montpellier-I, and \\ fLaboratoire de Physique Théorique et Astroparticules, CNRS UMR5207, Université Montpellier-II, Montpellier, France
}

\section{Key Words}

Deep brain stimulation - Dystonodyskinetic syndromes • Globus pallidus internus $\cdot$ Stereotactic model $\cdot$ Electrode design

\begin{abstract}
In nearly all deep brain stimulation (DBS) applications, the same quadripolar electrode design is used for different anatomical targets even if shape and volume differences exist between nuclei. Taking into account the electrode location within the internal globus pallidus (GPi) and the size of the $\mathrm{GPi}, 2$ electrodes were designed in order to improve the therapeutic benefit, to minimize side effects from DBS and to obtain a more homogeneous electric field distribution. The electrodes were evaluated numerically by using a stereotactic model measuring the correlation between the electric field and the GPi. The model was applied to 26 dystonodyskinetic patients who underwent surgery for a bilateral lead implantation into the posteroventral part of the $\mathrm{GPi}$. The designed electrodes produced a more homogeneous distribution of the electric field than the quadripolar electrode.

Copyright $\odot 2010$ S. Karger AG, Basel
\end{abstract}

\section{Introduction}

Deep brain stimulation (DBS) of the basal ganglia has become an alternative to surgical lesions for the treatment of several neurological disorders. Continuous highfrequency stimulation has proved effective in the treatment of essential tremor [1], Parkinson's disease [2] and dystonodyskinetic syndromes [3]. Numerous studies have examined the use of DBS in treating other neurological disorders $[4,5]$ as well as psychiatric disorders $[6$, 7]. Indications for DBS continue to expand. Depending on the symptoms, different anatomical targets such as the internal globus pallidus (GPi), the subthalamic nucleus (STN) or the ventral intermediate nucleus of the thalamus are chosen for stimulation. In nearly all DBS applications, the same electrode design is used even though differences in shape and volume exist between nuclei. The most widely used electrode is a quadripolar DBS electrode with cylindrical contacts (four contacts numbered from 0 to $3 ; 0=$ lower contact, $3=$ upper contact; contact height: $1.5 \mathrm{~mm}$; electrode diameter: $1.27 \mathrm{~mm}$; model 3389; Medtronic, Boulogne-Billancourt, France). Several studies have examined the effect of electrode design on the volume of tissue activated [8], the electrical distribution and the impedance [9]. The spatial distribution of the electric field is modified by varying the electrode geometry, in particular the aspect ratio (diameter/height)

Prof. Philippe Coubes

Unité de Recherche sur les Mouvements Anormaux, Hôpital Gui-de-Chauliac

80 , avenue Augustin-Fliche

FR-34295 Montpellier Cedex 5 (France)

Tel./Fax +3346733 74 64, E-Mail p-coubes@chu-montpellier.fr 
[8]. Future development and technology should include an electrode design that caters to the morphology and the anatomy of the target.

In our population of dystonodyskinetic patients treated with GPi electrode implantation, one or two contacts are sufficient to obtain an optimal clinical effect. The neurostimulators are initially set with one contact (contact 1 positioned in the target) as the cathode. The settings are adapted according to the clinical evolution of each patient. Several months later, if the patient's symptoms are not completely controlled by stimulation through a single contact, contact 2 is also activated. The activation of a supplementary contact or an increase in voltage does not guarantee the disappearance of the residual signs. In several patients, the addition of a second pair of leads within the sensorimotor GPi completed the initially incomplete response, certainly due to a somatotopic organization. However, in this situation, the current limitations of the DBS system necessitate the use of four Soletra internal pulse generators (IPG) or two double-entry IPG; these are large and not suitable for children. In order to improve the therapeutic benefit, to minimize side effects from DBS and to obtain a more homogeneous electric field distribution, we propose to consider a new design for the electrode.

The first step in designing a theoretically optimal DBS electrode for the GPi was to develop a three-dimensional (3-D) model allowing the calculation and visualization of the correlation between the electrode, the electrical distribution (isopotential, isofield and current density) and the GPi. First we developed a 3-D static model of the electrical distribution around the electrodes. Secondly, a stereotactic model of the GPi was developed by directly monitoring stereotactic points of the GPi borders on MRI according to our surgical protocol [10]. This technique makes allowance for individual structural variations between patients. For each patient, as the surgical space was stereotactically defined, a coregistration of the models was performed. Taking into account the GPi volume and the electrode length within the GPi, two electrodes were designed with the same diameter as the quadripolar electrode: a single-contact electrode (contact height of $5 \mathrm{~mm}$ ) and a double-contact electrode (contact height of $2.5 \mathrm{~mm}$, spaced by $0.5 \mathrm{~mm}$ ).

The electrodes were then numerically evaluated by applying the model to a reference population of 26 patients in whom DBS greatly improved primary generalized dystonodyskinesia, and who underwent a bilateral lead implantation in the posteroventral GPi. The stimulated volume, the impedance and the distribution of the electric field were studied.

\section{Materials and Methods}

Calculation of Isofield Distribution: Theoretical Model

A stereotactic model [11] of the in vivo stimulation system was developed, including the quadripolar electrode (model 3389; Medtronic) and the IPG (models 7424, 7425 and 7426; Medtronic).

The electrode model was positioned in a homogeneous cylinder (radius: $40 \mathrm{~mm}$; height: $80 \mathrm{~mm}$ ) with an isotropic resistance representing the brain tissue in the vicinity of the stimulating electrode. The borders of the cylinder were considered to be insulators in the bipolar mode. In the monopolar mode, the IPG was modelled as an additional perfect conductor disc placed at the bottom of the cylinder, with a radius of $20 \mathrm{~mm}$. The distribution of the potential $\mathrm{U}(\mathrm{r}, \mathrm{z})$ was determined as the solution of the Laplace equation where $r$ represents the radius and $\mathrm{z}$ the distance in height from the middle of the lead. The Laplace equation was reduced in cylindrical coordinates according to the axial symmetrical model:

$$
\frac{1}{r} \frac{\partial}{\partial r}\left(r \frac{\partial U}{\partial r}\right)+\frac{\partial^{2} U}{\partial z^{2}}=0
$$

Initial conditions were imposed for the calculation. The potential at the surfaces of the activated contacts corresponded to the programmed voltage and depended on the chosen stimulation mode. The non-stimulated contacts were considered to be electrical conductors that can present a potential different from 0 . To solve the equation, a finite-difference method was used. The electric field and the current density distribution were deduced from the potential.

\section{Stereotactic GPi Model}

The stereotactic protocol used for anatomic target localization in our centre [10] involves a pre- and a postoperative MRI under general anaesthesia. The stereotactic MRI enables a selection of stereotactic points along the GPi margins in the axial, sagittal and coronal planes. This is performed by the neurosurgeon, who identifies points (total points: approx. 50) of highest contrast confirmed as being located exactly at the edge of the targeted structure (fig. 1). The coordinates of each point are expressed in the Leksell reference system, without reference to an atlas. These points, called 'points of certainty' (C points), allow a 3-D mathematical reconstruction of the GPi, using a linear combination of radial basis functions [12], a mathematical method for building the GPi contours by determining an implicit equation of the surface formed by scattered points [11].

\section{Hardware and Software}

The theoretical models were implemented using a C/OpenGL (Open Graphics Library) computer programme that applies the marching cubes algorithm of Lorensen and Cline [13]. OpenGL is a standard specification defining a cross-language for writing applications that produces 2-D and 3-D computer graphics. The marching cubes algorithm of Lorensen and Cline is an algorithm to extract surface information from a 3-D field of values. This algorithm produces a triangle mesh by computing isosurfaces from discrete data.

As the surgical space was stereotactically defined, the models were coregistered for each patient. Two steps were required to cor- 
relate the anatomical information with the electrical distribution in a given patient. Firstly, $\mathrm{C}$ points and target coordinates including the trajectory angles were entered into the software. Secondly, the physician defined the electrical parameters of the electrode, recorded from the IPG, in order to visualize, manipulate and measure the correlation between the electrical distribution and the GPi anatomy of the patient. The software displayed an interface where the electrical settings and the geometry of the electrode could be changed. It was possible to extrapolate the line representing a specific isofield line (ISF) value (or isopotential value or current density value) from a family of lines (from 0 to $1 \mathrm{~V} / \mathrm{mm}$ ) and to calculate its volume and surface. It was also possible to display and calculate the surface and volume of both the GPi and the intersection between the isofield distribution and the $\mathrm{GPi}$, i.e. the intersection between the volume of each ISF value and the GPi volume.

\section{Statistical Analysis}

The descriptive analysis was performed using the mean \pm SD for the quantitative variables. Discrete variables were summarized as absolute numbers and percentages. For the precise assessment of the therapeutic efficacy, each patient was evaluated, preand postoperatively, using both modes (motor and disability scores) of the Burke-Marsden-Fahn Dystonia Rating Scale at predetermined intervals. Improvement was expressed as a percentage of the maximal possible gain [(Preoperative score - Postoperative score)/Preoperative score] $\times 100$. Because of the size of the group and the non-normal distributions, the comparisons were performed using the Mann-Whitney U test. The statistical analyses were performed using the free software R.

\section{Patient Population}

In order to design an appropriate electrode for the GPi, the model was applied to data recorded from the case studies of 26 right-handed patients with primary dystonodyskinetic syndromes at a steady state ( 13 female and 13 male; mean age: $22.3 \pm$ 14.2 years; mean age at onset: $12.2 \pm 7.8$ years), in particular preand postoperative MRI data indicating anatomical information, target coordinates and trajectory angles. The patients were treated with bilateral posteroventral GPi implantation. Movement disorders were pre- and postoperatively assessed by a neurologist and a neurosurgeon trained in movement disorders (L.C. and P.C.) at predetermined intervals using both modes of the BurkeMarsden-Fahn Dystonia Rating Scale. The 26 patients improved respectively to $90.1 \pm 10 \%$ and $79.1 \pm 29.9 \%$ of the motor and disability scores. The mean follow-up time was $4.4 \pm 2$ years.

All the patients received high-frequency $(130 \mathrm{~Hz})$ neurostimulation with a pulse width of $450 \mu \mathrm{s}$ in monopolar (26 GPi) and double monopolar mode (26 GPi). The amplitude was set between $0.5 \mathrm{~V}$ and a maximum of $2.2 \mathrm{~V}$, according to the clinical response and stimulation mode.

\section{Electrode Design}

The electrode length within the GPi for each patient was measured (fig. 2) in order to design an appropriate electrode specific to the GPi. The mean length of the electrode within the GPi was 6.4 $\pm 1.3 \mathrm{~mm}$ (mean electrode length for the right GPi: $6.2 \pm 1.9 \mathrm{~mm}$; left GPi: $6.7 \pm 1.3 \mathrm{~mm}$ ). To cater to the mean length including the SD, we chose to design 2 electrodes (fig. 3): a single-contact electrode (electrode 1; diameter $=1.27 \mathrm{~mm}$; height of the

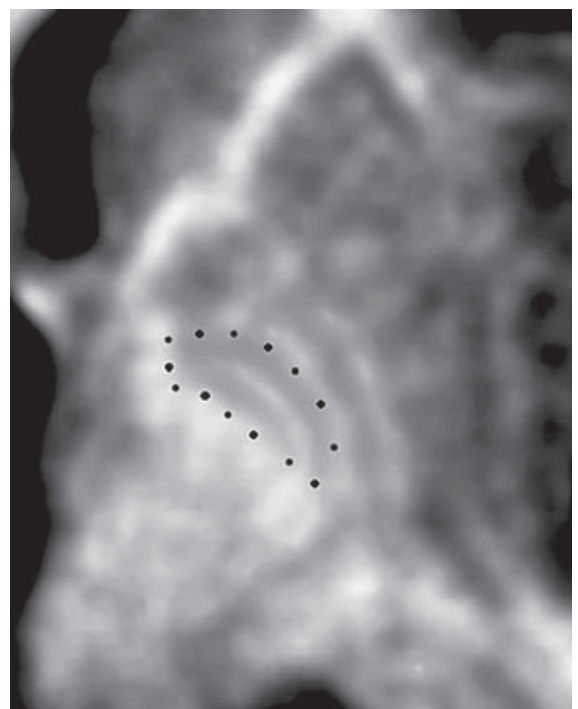

Fig. 1. The border points of the GPi superimposed on the MRI.

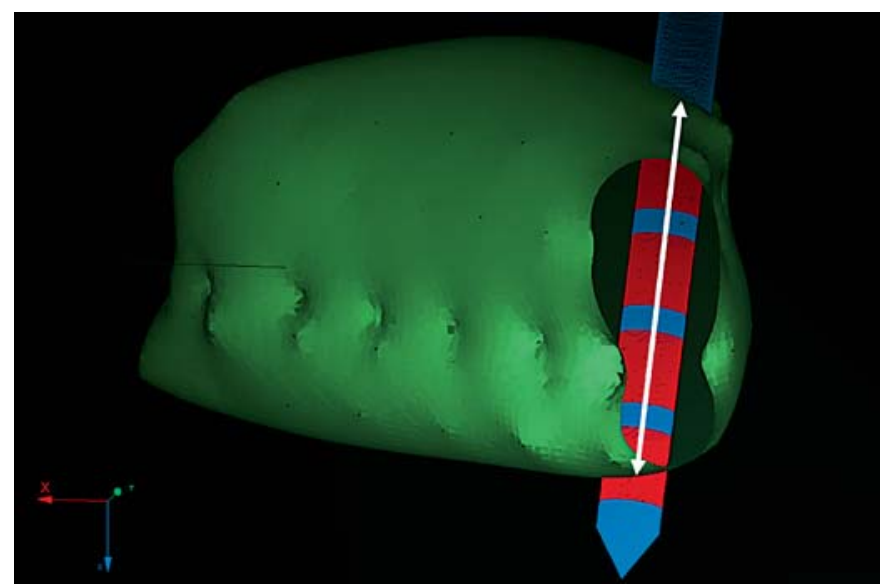

Fig. 2. Illustration of a quadripolar electrode within the GPi of a patient. The electrode lengths within the GPi (two-headed arrow) were measured for the 26 patients. Bottom left corner: Cartesian axes $(\mathrm{x}, \mathrm{y}, \mathrm{z})$.

contact $=5 \mathrm{~mm}$ ) and a double-contact electrode (electrode 2 ; diameter $=1.27 \mathrm{~mm}$; height of each contact $=2.5 \mathrm{~mm}$; space between contacts $=0.5 \mathrm{~mm}$; contact 1 is the lower and contact 2 the upper contact).

Taking into account the patient's electrical parameters associated with the quadripolar electrode, the intersection between the volume of the selected ISF values [0.1 $\left(\mathrm{ISF}_{0.1}\right), 0.2\left(\mathrm{ISF}_{0.2}\right)$ and 0.4 $\left.\mathrm{V} / \mathrm{mm}\left(\mathrm{ISF}_{0.4}\right)\right]$ and the volume of the GPi was calculated for each patient. This intersection gave a volume stimulated by each ISF value.

The trial electrodes were positioned in the GPi with the target coordinates and the trajectory angles provided by the postoperative 

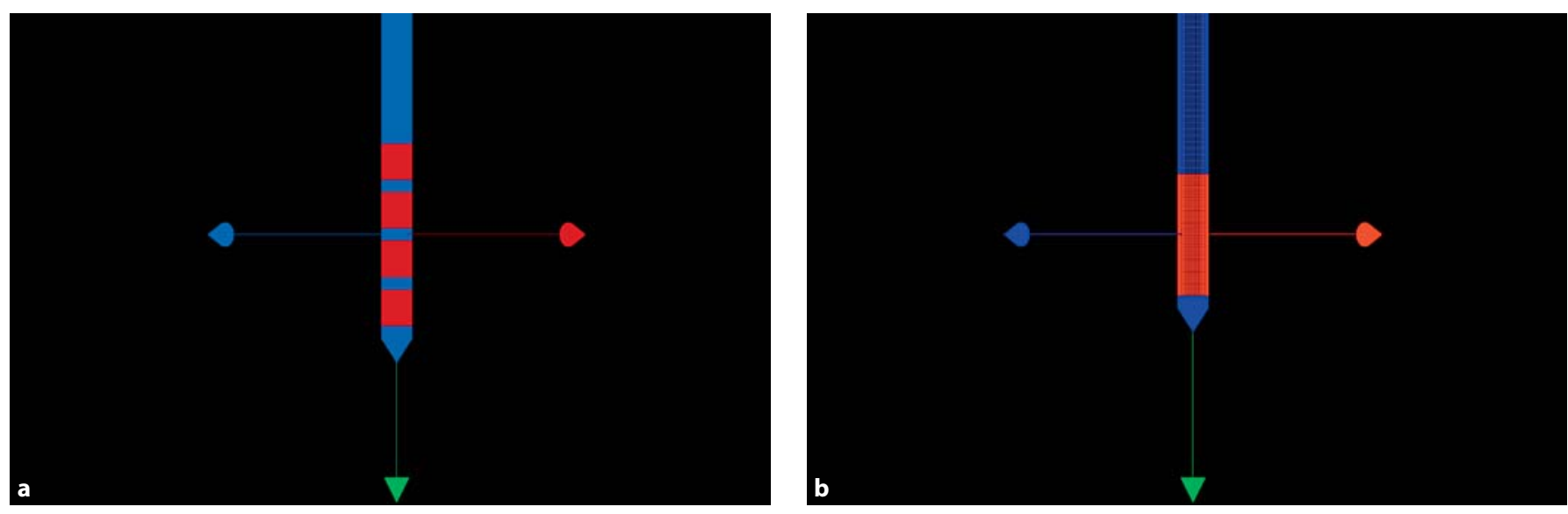

Fig. 3. Electrode illustration. $\operatorname{Red}=\mathrm{x}$-axis; green $=\mathrm{y}$-axis; blue $=$ z-axis. a The widely used quadripolar DBS electrode (model 3389; Medtronic) with 4 cylindrical contacts. Contact height: $1.5 \mathrm{~mm}$, spaced by $0.5 \mathrm{~mm}$. Diameter: $1.27 \mathrm{~mm}$. b Electrode 1. Single-contact electrode. Diameter: $1.27 \mathrm{~mm}$. Contact height: $5 \mathrm{~mm}$. c Electrode 2. Double-contact electrode. Diameter: $1.27 \mathrm{~mm}$. Contact height: $2.5 \mathrm{~mm}$, spaced by $0.5 \mathrm{~mm}$. Contact 1 refers to the lower contact and contact 2 to the upper contact.

Table 1. Mean stimulated volume $\pm \mathrm{SD}$ of GPi by $\mathrm{ISF}_{0.1}, \mathrm{ISF}_{0.2}$ and $\operatorname{ISF}_{0.4}\left(\mathrm{~mm}^{3}\right)$

\begin{tabular}{llll}
\hline & $\mathrm{ISF}_{0.1}$ & $\mathrm{ISF}_{0.2}$ & $\mathrm{ISF}_{0.4}$ \\
\hline Right GPi & $84.3 \pm 32.8$ & $35.4 \pm 14.1$ & $12.2 \pm 4.1$ \\
Left GPi & $86.5 \pm 26.3$ & $35.4 \pm 13.2$ & $12.7 \pm 4.4$ \\
Total & $85.4 \pm 29.4$ & $35.4 \pm 13.5$ & $12.4 \pm 4.2$ \\
\hline
\end{tabular}

The volumes were determined by calculating the intersection between the volume of each ISF value and the volume of the GPi for each patient.

MRI. The target was aligned with the middle of the single contact for electrode 1 , and between contacts 1 and 2 for electrode 2 .

The electrical settings of the trial electrodes were manipulated in order to reach the same stimulated volume by the $\mathrm{ISF}_{0.1}, \mathrm{ISF}_{0.2}$ and $\mathrm{ISF}_{0.4}$ values as the quadripolar electrode. The volume of the ISF $_{0.2}$ generated by the electrodes was calculated for different configurations at a constant voltage of $1.5 \mathrm{~V}$.

\section{Results}

\section{Intersection between GPi Volume and ISF Volumes}

Electric field distribution was correlated with the 26 patients' anatomical information using the pre- and postoperative stereotactic MRI (fig. 4).

The mean volume of the GPi was $528.1 \pm 87.4 \mathrm{~mm}^{3}$ (mean volume of the right GPi: $539.9 \pm 86.7 \mathrm{~mm}^{3}$; left GPi: $515.7 \pm 88.1 \mathrm{~mm}^{3}$ ). Three electric field values were studied more specifically: $\mathrm{ISF}_{0.1}, \mathrm{ISF}_{0.2}$ and $\mathrm{ISF}_{0.4}$.

At a steady state, the mean GPi volume stimulated by the 3 ISF, i.e. the intersection between the volume of the GPi and the volume of each ISF, is shown in table 1.

For the monopolar-mode population, the mean voltage used by the quadripolar electrode was $1.5 \pm 0.3 \mathrm{~V}$ in the right GPi and $1.57 \pm 0.34 \mathrm{~V}$ in the left GPi. In order to reach the same stimulated volume for the selected ISF, the voltage of electrodes 1 and 2 was adapted for each patient (table 2). Compared to the mean voltages required for the quadripolar electrode, less voltage was necessary 

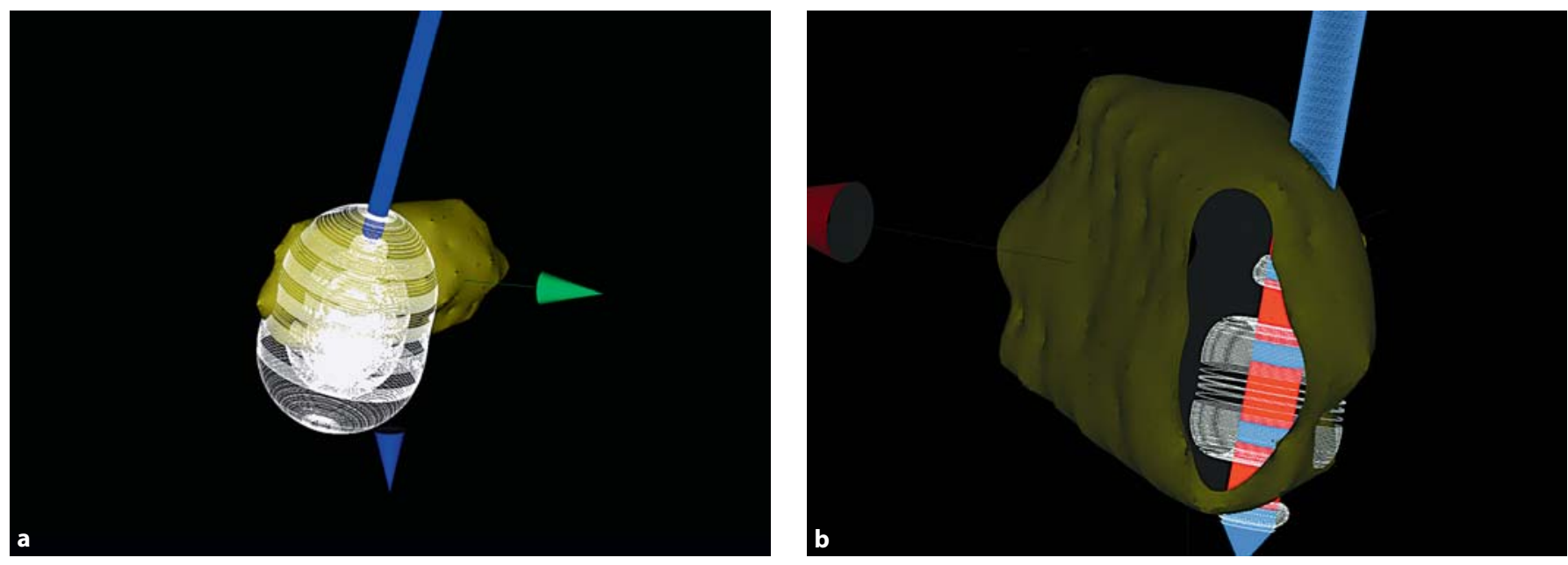

Fig. 4. Correlation between anatomical and electric field data in an illustrative case showing marked improvement with posteroventral GPi DBS. a All the isofield lines from 0 to $1 \mathrm{~V} / \mathrm{mm}$ in increments of $0.025 \mathrm{~V} / \mathrm{mm}$ in the GPi volume. $\mathbf{b}$ The isofield line of $0.2 \mathrm{~V} / \mathrm{mm}$ is isolated.

Table 2. Voltage increase required to reach the same stimulated volume by quadripolar electrode or each ISF value (V)

\begin{tabular}{|c|c|c|c|c|c|c|}
\hline & \multicolumn{6}{|l|}{ Voltage } \\
\hline & \multicolumn{2}{|l|}{$\mathrm{ISF}_{0.1}$} & \multicolumn{2}{|l|}{$\mathrm{ISF}_{0.2}$} & \multicolumn{2}{|l|}{$\mathrm{ISF}_{0.4}$} \\
\hline & right & left & right & left & right & left \\
\hline \multicolumn{7}{|l|}{ Monopolar mode } \\
\hline Quadripolar electrode & $1.5 \pm 0.3$ & $1.57 \pm 0.34$ & $1.5 \pm 0.29$ & $1.57 \pm 0.34$ & $1.5 \pm 0.29$ & $1.57 \pm 0.34$ \\
\hline Electrode 1 & $0.94 \pm 0.17$ & $0.99 \pm 0.19$ & $1.03 \pm 0.13$ & $1.07 \pm 0.17$ & $1.48 \pm 0.25$ & $1.4 \pm 0.16$ \\
\hline Electrode 2 & $1.2 \pm 0.25$ & $1.29 \pm 0.28$ & $1.18 \pm 0.2$ & $1.15 \pm 0.26$ & $1.3 \pm 0.19$ & $1.39 \pm 0.23$ \\
\hline \multicolumn{7}{|l|}{ Double monopolar mode } \\
\hline Quadripolar electrode & $1.49 \pm 0.26$ & $1.5 \pm 0.31$ & $1.49 \pm 0.26$ & $1.5 \pm 0.31$ & $1.49 \pm 0.26$ & $1.5 \pm 0.31$ \\
\hline Electrode 1 & $1.28 \pm 0.23$ & $1.28 \pm 0.25$ & $1.28 \pm 0.27$ & $1.3 \pm 0.25$ & $1.48 \pm 0.18$ & $1.5 \pm 0.24$ \\
\hline Electrode 2 & $1.25 \pm 0.21$ & $1.26 \pm 0.25$ & $1.23 \pm 0.27$ & $1.3 \pm 0.25$ & $1.47 \pm 0.18$ & $1.5 \pm 0.24$ \\
\hline
\end{tabular}

for the trial electrodes to reach the same stimulated volumes at $\mathrm{ISF}_{0.1}$ and $\mathrm{ISF}_{0.2}$. A significant difference $(\mathrm{p}<$ 0.001) was found for the $\mathrm{ISF}_{0.1}$ and $\mathrm{ISF}_{0.2}$. No significant difference was found for the $\operatorname{ISF}_{0.4}(\mathrm{p}=0.203)$.

For the double-monopolar-mode population, the mean voltage used by the quadripolar electrode was 1.49 $\pm 0.26 \mathrm{~V}$ in the right GPi and $1.5 \pm 0.31 \mathrm{~V}$ in the left GPi. Comparing the mean voltages of the quadripolar electrode with the trial electrodes, a significant difference $(\mathrm{p}<0.001)$ was found for the $\mathrm{ISF}_{0.1}$ and $\mathrm{ISF}_{0.2}$. No significant difference was found for the $\operatorname{ISF}_{0.4}(\mathrm{p}=$ $0.587)$.

\section{Electric Field Distribution}

The visualization of the isofield lines (fig. 5) showed a more homogeneous distribution of the electric field for the trial electrodes compared to the quadripolar electrode. The volume of the $\mathrm{ISF}_{0.2}$ generated by the electrodes was calculated for different configuration modes with a constant voltage of $1.5 \mathrm{~V}$ (table 3 ). An increase in electrode surface area decreased the impedance. The $\mathrm{ISF}_{0.2}$ obtained a higher volume with the single-contact electrode $\left(64 \mathrm{~mm}^{3}\right)$ and the double monopolar configuration with electrode $2\left(64.1 \mathrm{~mm}^{3}\right)$. The same configurations had smaller theoretical impedances. The quadripolar electrode had smaller volumes.

Stereotact Funct Neurosurg 2010;88:129-137 

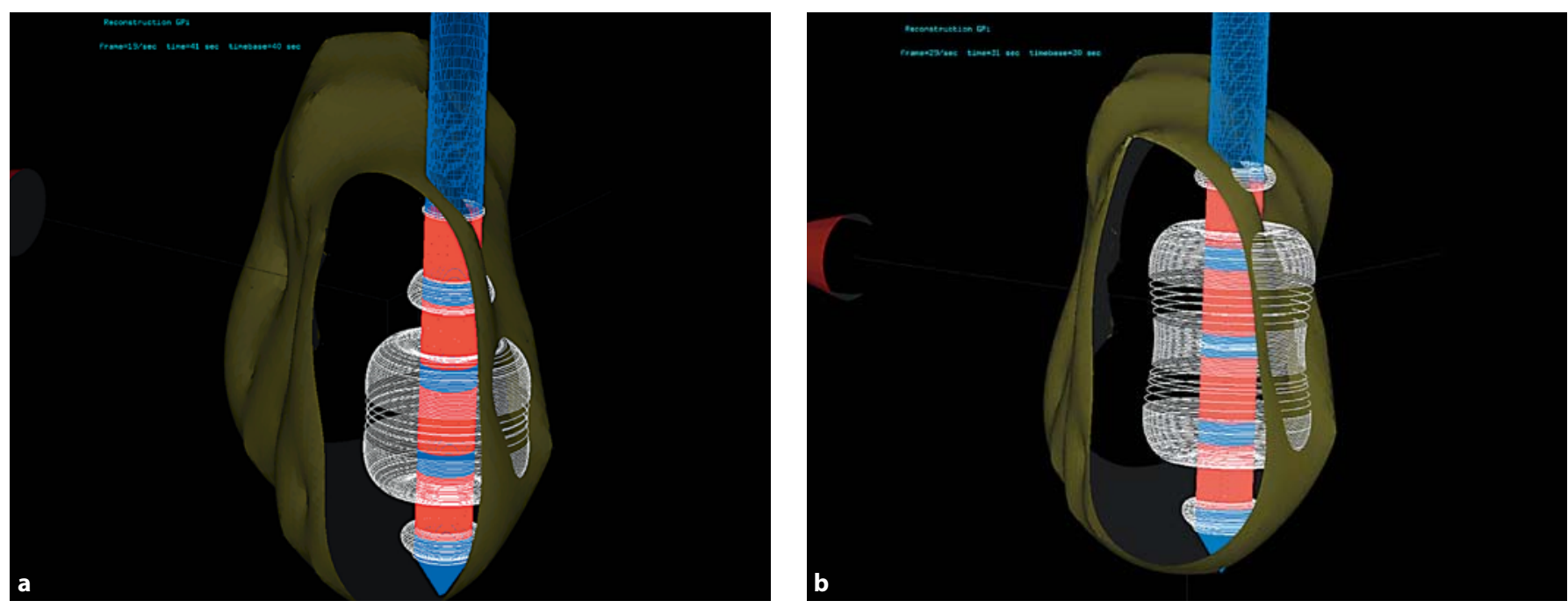

Fig. 5. The $0.2-\mathrm{V} / \mathrm{mm}$ isofield line distributions with a voltage of $1.5 \mathrm{~V}$ for different stimulation modes within the GPi. Quadripolar electrode with contact 1 as the cathode (a), contacts 1 and 2 as the cathodes (b), and contact 1 as the cathode and contact 2 as the anode (c).

\section{Discussion}

In nearly all DBS applications, the same electrode design is used even though shape and volume differences exist between targets. The aim of this study was to design two electrodes for specific use in the GPi, and to evaluate them quantitatively, using a stereotactic model to visualize and measure the distribution of the electric field around each electrode when implanted. Indeed, the DBS electrode contact geometry had been shown to influence the volume of the electric field by Butson and McIntyre [8], who proposed an electrode design for the ventral intermediate nucleus of the thalamus.

\section{Study Limitations}

One limitation of this study is the use of a homogeneous and isotropic model. Many groups [14-16] use anisotropic brain models which take into account the white/grey matter distribution and fibre direction, using diffusion tensor imaging. Tissue inhomogeneity and isotropy such as small lacunar cavities [16] can modify the shape of the electric field distribution. Notably, Butson et al. [15] developed a model of the STN for Parkinson's disease showing how the surrounding structures may influence the shape of the electric field. This approach is promising because it takes into account the heterogeneity of the brain. Up to now, even though highly specific, these models have been difficult to apply to neurosurgical and clinical practice where individual brain 

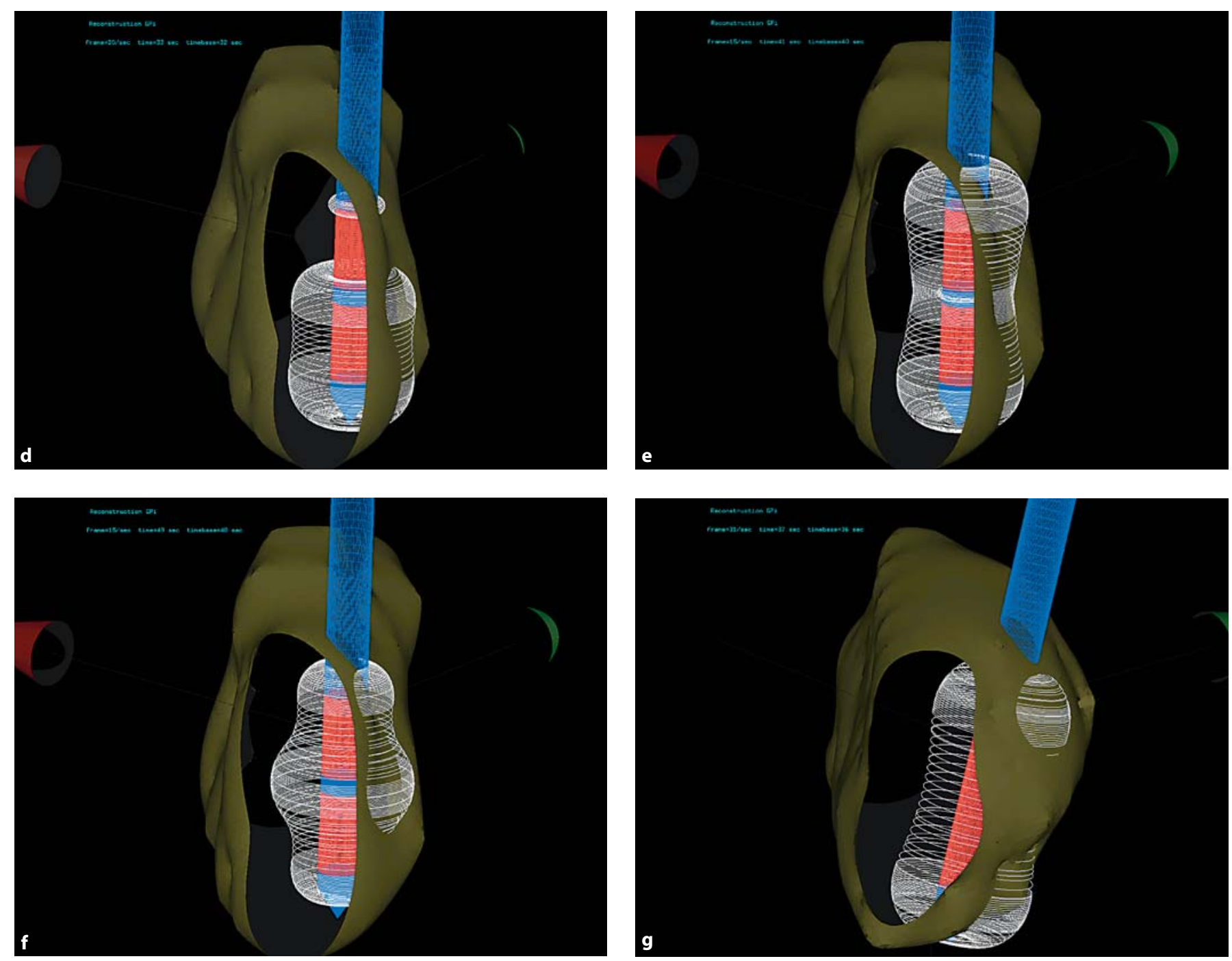

Fig. 5. The $0.2-\mathrm{V} / \mathrm{mm}$ isofield line distributions with a voltage of $1.5 \mathrm{~V}$ for different stimulation modes within the GPi. Double-contact electrode in monopolar (d), double monopolar (e) and bipolar (f) configuration. Singlecontact electrode with the only contact as the cathode $(\mathbf{g})$.

anatomy is the most important parameter. The precision of the surgical procedure is an issue of major importance due to the fact that lead insertion with $0.5 \mathrm{~mm}$ accuracy is needed to hit the target. Diffusion tensor imaging is not currently appropriate due to the limited image resolution. An accurate measurement of small structures or interfaces between structures like GPi/external GP requires high spatial resolution, but high-resolution submillimetre diffusion tensor imaging cannot currentlybe achieved. In order to create a model using diffusion tensor imaging, it would be necessary to use multiple image coregistration and atlas representations of the patient, involving spatial variability [17], which is not feasible during surgery because of the accuracy required and the time limitations in surgery.

The efficacy and reproducibility of this therapy are based on a concrete and precise delineation of the GPi limits [18]. In line with our experience in movement disorder surgery, based on direct MRI targeting of the GPi, the $C$ point method was developed in order to maximally reduce the subjectivity of target localization [11]. Our stereotactic protocol for anatomic target localization involves a pre- and a postoperative 1.5-tesla MRI (slice thickness of $1 \mathrm{~mm}$ for both $\mathrm{T}_{1^{-}}$and $\mathrm{T}_{2}$-weighted images) 
Table 3. Volume of $\mathrm{ISF}_{0.2}$ generated by each electrode calculated for different configuration modes at the same voltage of $1.5 \mathrm{~V}$

\begin{tabular}{llc}
\hline Electrode configuration & $\begin{array}{l}\text { Volume } \\
\mathrm{mm}^{3}\end{array}$ & $\begin{array}{l}\text { Theoretical } \\
\text { impedance, } \Omega\end{array}$ \\
\hline $\begin{array}{l}\text { Quadripolar electrode } \\
\text { 0m00 }\end{array}$ & 29.4 & 1,185 \\
$\quad$ 0mm0 & 48.1 & 811.6 \\
0mp0 & 31.2 & $1,478.8$ \\
Electrode 1 & & \\
$\quad$ m & 64 & 687.1 \\
Electrode 2 & & \\
$\quad$ m0 & 43.9 & 936.1 \\
mm & 64.1 & 645.1 \\
mp & 49.9 & $1,173.9$ \\
\hline
\end{tabular}

$\mathrm{m}=$ Minus; $\mathrm{p}=$ plus.

under general anaesthesia. This procedure enables the selection of stereotactic points of the GPi borders directly on MRI in the axial, sagittal and coronal planes. This is performed by visualizing points of highest contrast located exactly at the edge of the targeted structure. Especially in the case of a disorder secondary to a lesion, the internal medullary lamina can be irregularly modified and the level of contrast between structures may be particularly low. The originality of the method proposed is that $C$ points are defined under visual control by the neurosurgeon, taking into account interindividual variation including the variation in internal medullary laminae in several cases. The neurosurgeon decides on the target limits and pinpoints with certitude the pixel considered to be at the interface [11].

For the purposes of this study, a homogeneous and isotropic model is acceptable, given the low density of neurons in the GPi compared with the STN [19]. Furthermore, the grey matter of the nervous system is isotropic with a conductivity of approximately $0.2 \mathrm{~S} / \mathrm{m}$ [20-22]. The white matter, composed of fibres, is anisotropic with a higher conductivity $(1 \mathrm{~S} / \mathrm{m})$ in the direction parallel to the fibres than in the direction perpendicular to the fibres $(0.1 \mathrm{~S} / \mathrm{m})$ [23].

In conclusion, an activated electrode contact in the white matter will provide a greater diffusion of the electrical potential and of the electric field than a contact localized in the grey matter (GPi). Also, we published studies in which we demonstrated that, in patients with maximum benefits, the optimal volume necessary for obtaining the effect did not cover the entire motor GPi
( $\mathrm{ISF}_{0.2}$ within the GPi) and then, within the isotropic grey matter $[24,25]$. This allowed the study to be performed in the most controlled environment possible.

\section{Electrode Design Specific to GPi}

According to Ohm's law, the impedance decreases by increasing the surface area of the electrode contact, as shown by Wei and Grill [9], the impedance being inversely related to the electrode area. Furthermore, our results show that the volume stimulated by the trial electrodes requires less voltage than the quadripolar electrode currently used, and that a more homogeneous distribution of the electric field is provided by the trial electrode. Consequently, the trial electrodes optimize the stimulated volume of the GPi and could therefore increase the therapeutic benefits and reduce side effects. This is an issue of major interest, given that increasing the number of activated contacts and/or the voltage does not allow an additional improvement and the control of all the signs in patients responding to the therapy.

Also, even if the efficacy of GPi DBS is maintained over time, in our experience, a reoccurrence of the signs and/or an occurrence of new signs can appear. However, in several patients, the addition of a second electrode implantation in the sensorimotor GPi has been found to complete an initially incomplete response. The addition of a second electrode seems to be linked with a somatotopic organization within the GPi [26]. At the initial procedure, multiple electrode implantations into the GPi can be required in order to control all the signs of the disease and to avoid another surgery for reimplantation. In this situation, the current limit of the DBS system imposes the use of four Soletra IPG or two double-entry IPG, which are large and not suitable for children. The trial electrodes could overcome this limitation. The double-contact electrode proposed in this study seems to be the more appropriate of the two for GPi stimulation. Firstly, even if stimulation is more effective near the negative pole than the positive pole, the double-contact electrode allows a bipolar configuration, used by the clinicians to limit edge effects. Furthermore, earlier reports [27-29] have shown that the most effective positions of the lead are in the posterior, medial and ventral parts of the GPi, shown to be the sensorimotor area, and close to the output pathway, i.e. the ansa lenticularis. If these fibres must be activated to obtain an optimum therapeutic effect, the double-contact electrode could provide a stronger and more homogeneous stimulation of this region. The electric field generated by the single-contact electrode (fig. 5) spreads along the side of the contact, stimulating the dorsal part of the 
GPi described as being the associative area [30,31]; therefore, it is not effective. To confirm these initial findings and to allow a better understanding of them, further data including patient trials in association with the biomedical industry are necessary for clinical validation.

\section{Conclusion}

The geometrical characteristics of a DBS electrode are an issue of major importance due to the differences between targets and the clinical evolution in dystonodyskinetic patients. A double contact with a height of $2.5 \mathrm{~mm}$ seems to be more appropriate for posteroventral GPi stimulation. This electrode allows a decrease in imped- ance and a homogeneous distribution of the electric field. Furthermore, less voltage is needed to reach the same stimulated volume compared with the quadripolar electrode. Further studies with the biomedical industry must be conducted in order to manufacture the electrode for clinical validation. The progression to its usage in surgery should not necessitate a specific evaluation, as the new electrode will be closely similar to the current electrode in terms of material.

\section{Acknowledgements}

This study was supported by grants from the Wyeth Foundation and from IBM Montpellier, Products and Solutions Support Center (PSSC).

\section{References}

$\checkmark 1$ Benabid AL, et al: Long-term suppression of tremor by chronic stimulation of the ventral intermediate thalamic nucleus. Lancet 1991; 337:403-406

$\checkmark 2$ Pollak P, et al: Treatment results: Parkinson's disease. Mov Disord 2002;17(suppl 3):S75S83.

-3 Coubes P, et al: Electrical stimulation of the globus pallidus internus in patients with primary generalized dystonia: long-term results. J Neurosurg 2004;101:189-194.

4 Wishart HA, et al: Chronic deep brain stimulation for the treatment of tremor in multiple sclerosis: review and case reports. J Neurol Neurosurg Psychiatry 2003;74:1392-1397.

$\checkmark 5$ Hodaie M, et al: Chronic anterior thalamus stimulation for intractable epilepsy. Epilepsia 2002;43:603-608.

6 Nuttin BJ, et al: Electrical stimulation of the anterior limbs of the internal capsules in patients with severe obsessive-compulsive disorder: anecdotal reports. Neurosurg Clin N Am 2003; 14:267-274.

7 Mayberg HS, et al: Deep brain stimulation for treatment-resistant depression. Neuron 2005;45:651-660.

-8 Butson CR, McIntyre CC: Role of electrode design on the volume of tissue activated during deep brain stimulation. J Neural Eng 2006;3:1-8.

9 Wei XF, Grill WM: Current density distributions, field distributions and impedance analysis of segmented deep brain stimulation electrodes. J Neural Eng 2005;2:139-147.

$\checkmark 10$ Coubes P, et al: Deep brain stimulation for dystonia: surgical technique. Stereotact Funct Neurosurg 2002;78:183-191.

11 Vasques X, et al: Stereotactic model of the electrical distribution within the internal globus pallidus during deep brain stimulation. J Comput Neurosci 2009;26:109-118.
12 Morse B, et al: Interpolating implicit surfaces from scattered surface data using compactly supported radial basis functions. Proceedings of the International Conference on Shape Modeling and Applications 2001. Washington, IEEE Computer Society, 2001, pp 89-98.

13 Lorensen W, Cline H: Marching Cubes: a high-resolution 3D surface construction algorithm. Computer Graphics 1987;21:163169.

14 McIntyre CC, et al: Electric field and stimulating influence generated by deep brain stimulation of the subthalamic nucleus. Clin Neurophysiol 2004;115:589-595.

15 Butson CR, et al: Patient-specific analysis of the volume of tissue activated during deep brain stimulation. Neuroimage 2007;34: 661-670.

16 Aström M, et al: The effect of cystic cavities on deep brain stimulation in the basal ganglia: a simulation-based study. J Neural Eng 2006;3:132-138.

17 Vayssière $\mathrm{N}$, et al: Comparison of atlas- and magnetic resonance imaging-based stereotactic targeting of the globus pallidus internus in the performance of deep brain stimulation for treatment of dystonia. J Neurosurg 2002;96:673-679.

18 Vayssière $\mathrm{N}$, et al: Magnetic resonance imaging stereotactic target localization for deep brain stimulation in dystonic children. Neurosurg 2000;93:784-790.

19 Yelnik J: Functional anatomy of the basal ganglia. Mov Disord 2002;17(suppl 3):S15-S21.

20 Kuncel AM, Grill WM: Selection of stimulus parameters for deep brain stimulation. Clin Neurophysiol 2004;115:2431-2441.

21 Li CH, Bak AF, Parker LO: Specific resistivity of the cerebral cortex and white matter. Exp Neurol 1968;20:544-557.
22 Ranck JB Jr: Specific impedance of rabbit cerebral cortex. Exp Neurol 1963;7:144-152.

23 Nicholson PW: Specific impedance of cerebral white matter. Exp Neurol 1965;13:386401 .

24 Vasques X, et al: Prognostic value of globus pallidus internus volume in primary dystonia treated by deep brain stimulation. J Neurosurg 2009;110:220-228.

25 Vasques X, et al: Factors predicting improvement in primary generalized dystonia treated by pallidal deep brain stimulation. Mov Disord 2009;24:846-853.

26 Vayssiere N, et al: Deep brain stimulation for dystonia confirming a somatotopic organization in the globus pallidus internus. J Neurosurg 2004;101:181-188.

27 Houeto JL, et al: Acute deep-brain stimulation of the internal and external globus pallidus in primary dystonia: functional mapping of the pallidum. Arch Neurol 2007;64: 1281-1286.

-28 Tisch S, et al: Effect of electrode contact location on clinical efficacy of pallidal deep brain stimulation in primary generalised dystonia. J Neurol Neurosurg Psychiatry 2007;78:1314-1319.

29 Laitinen LV, BergenheimAT, Hariz MI: Leksell's posteroventral pallidotomy in the treatment of Parkinson's disease. J Neurosurg 1992;76:53-61.

30 Karachi C, et al: Three-dimensional cartography of functional territories in the human striatopallidal complex by using calbindin immunoreactivity. J Comp Neurol 2002;450: 122-134.

31 Yelnik J, et al: A three-dimensional, histological and deformable atlas of the human basal ganglia. 1. Atlas construction based on immunohistochemical and MRI data. Neuroimage 2007;34:618-638. 